\title{
Chaos control in Shimizu Morioka system by Lie algebraic exact linearization
}

\author{
Mitul Islam • Bipul Islam • Nurul Islam
}

Received: 6 September 2013 / Revised: 17 November 2013 / Accepted: 7 December 2013 / Published online: 21 December 2013

(C) Springer-Verlag Berlin Heidelberg 2013

\begin{abstract}
Chaos in Shimizu-Morioka system is controlled by applying a Lie algebraic exact linearization technique. In this state space exact linearization method, a non-linear feedback control law is designed which induces a coordinate transformation thereby changing the original non-linear chaotic system into a linear controllable one. The controller is designed for an arbitrary output and the paper discusses the case of both linear and non-linear outputs. Necessary and sufficient conditions are derived for stabilization of the system to a point and onto a limit cycle. Numerical simulation has been conducted for all these aspects of the problem that conclusively establishes our analytical findings.
\end{abstract}

Keywords Exact linearization - Lie bracket - Lie derivative $\cdot$ Nonlinear feedback $\cdot$ Observer .

Shimizu-Morioka system $\cdot$ Limit cycle $\cdot$ Hopf bifurcation

\section{Introduction}

Existence of chaos in dynamical systems is an interesting phenomenon in physics, mathematics as well various engineering subjects. Controllability of the same as well as imple-

\author{
M. Islam ( $\square)$ \\ Department of Mathematics, Jadavpur University, \\ Kolkata 700032, India \\ e-mail: mitul.islam@gmail.com \\ B. Islam \\ Indian Statistical Institute, 203 B.T. Road, \\ Barrackpore, Kolkata 700108, India \\ e-mail: islam.bipul@gmail.com \\ N. Islam \\ Department of Mathematics, Ramakrishna Mission College \\ (Autonomous), Narendrapur, Kolkata 700103, India \\ e-mail:dr.i.nurul@gmail.com
}

menting control strategies to control the chaos has been a subject of interest and investigation for quite some time meriting publications that number in thousands. Various nonconventional engineering control methodologies have been proposed till date by experimental, theoretical physicists and mathematicians.

Open loop control, closed loop control, parametric entrainment control, also a 'mix and match' between these strategies are some of the well-known control methodologies for chaos control [1]. Apart from this, we have variations of feedback control, viz.-linear, non-linear and time-delayed feedback, adaptive algorithms, bang-bang control, being some of the popular choices for chaos control [2]. The stabilization of the system in these methods is either through use of Lyapunov functions or through linearization by using Taylor's theorem. While the first method gives global results, the second method of inexact linearization gives only the local results. An alternative approach is to transform the non-linear system to an equivalent linear system through invertible coordinate transformation. This method, though localized because the existence of the inverse function can only be guaranteed locally, is much more effective than the conventional inexact linearization methods. Feedback linearization or state space exact linearization is the most commonly used method for obtaining this goal [3-5]. First proposed in 1963, it involves an elaborate scheme that utilises Lie algebraic techniques for designing the required coordinate transformation. A closed loop control strategy is employed to make the system linearizable through coordinate transformation. Liqun and Yanzhu [6] and Alvarez [7] had used the state-space linearization method for controlling chaos in Lorenz system.

In this paper, our aim is to control the chaos in the Shimizu-Morioka(SM) system [8,9]. It is a Lorenz like chaotic system that captures the qualitative properties of 'Lorenz-ian' chaos within an algebraically simpler structure. 
Algebraic simplicity is due to the fact that it has six terms with two quadratic non-linearity while Lorenz system had seven terms with two quadratic non-linearity [10]. ShimizuMorioka system appears in the analysis of semi-conductor lasers and hence its control studies are of considerable importance. In our approach, the control is exercised on the second equation of the system and it's effect on the system structure is studied analytically as well as numerically. One of the most important results that has been obtained in this communication is the stabilization of the chaotic system to a limit cycle without using the conventional time-delayed feedback. The benefit of this approach is the scope of driving the system into a periodically varying state with bounded regular oscillations. Put in other words, the system attains a control goal that varies with time periodically. General feedback control mechanisms, including the feedback linearization, focuses on controlling the system to a control goal, which is actually the equilibrium point of the corresponding linear system. This paper covers both the situations of stabilization to an equilibrium point as well as onto a limit cycle. The paper also compares the effect of using linear and non-linear outputs on a feedback linearized system by discussing two separate cases for the same problem. Moreover, exploration of the parameter space numerically reveals that the dynamics of the controlled system cannot get anymore complex - the system either stabilizes to a point, or to a limit cycle, or the system remains unstable.

Section 2 gives an overview of the state space exact linearization method so as to enhance the readability of the following sections. Section 3 puts forth the Shimizu-Morioka system that will be controlled. Section 4 contains the main results of the paper. Theorems 1 and 2 provide the necessary and sufficient conditions for stabilizing the system to a point and onto a limit cycle respectively. Theorem 3 produces the controller that controls Shimizu-Morioka chaotic system for any given output function. Sections 4.1 and 4.2 illustrates the scheme with a linear and a non-linear output respectively. Both the sections contain respective numerical simulation results that support our analytical findings.

\section{Exact linearization using nonlinear feedback}

Feedback linearization is a technique of controlling a nonlinear dynamical system. This technique involves transformation of a given non-linear system into a linear system by using a suitable control input and by a change of state variables.

Let us consider a non-linear dynamical system as

$\dot{x}=f(x)$.

Its corresponding single input non-linear control system can be taken as $\dot{x}=f(x)+g(x) u$

where $x \in \mathbb{R}^{n}$, is the state vector; and $u \in \mathbb{R}$, is the control parameter. $f: \mathbb{R}^{n} \rightarrow \mathbb{R}^{n}$ and $g: \mathbb{R}^{n} \rightarrow \mathbb{R}^{n}$ are both smooth vector fields on $\mathbb{R}^{n}$.

Result The system (2) is called feedback linearizable in the domain $\Omega \subset \mathbb{R}^{n}$ if there exists a smooth reversible change of coordinates $z=T(x), x \in \Omega$ and a smooth transformation of feedback $v=\alpha(x)+\beta(x) u, x \in \Omega$, where $v \in \mathbb{R}$ is the new control if the closed loop system is linear [1].

Lie algebraic methods are used in this technique. For two vector fields $f(x)$ and $g(x)$, different order Lie brackets are denoted by the symbols as follows: $a d_{f}^{k} g(x)=$ $\left[f, a d_{f}^{k-1} g\right](x) ; k=1,2,3, \ldots$; with $a d_{f}^{0} g(x)=g(x)$, and each of $a d_{f}^{k} g(x) \in \mathbb{R}^{n}$ for $k=1,2,3, \ldots$.

If we write $a d_{f}^{k} g(x)=\left[\left(a d_{f}^{k} g(x)\right)_{1},\left(a d_{f}^{k} g(x)\right)_{2}, \ldots\right.$ $\left.\left(a d_{f}^{k} g(x)\right)_{n}\right]^{T}$; then $\left(a d_{f}^{k} g(x)\right)_{j}$ is computed by the formula:

$$
\begin{aligned}
\left(a d_{f}^{k} g(x)\right)_{j}= & \Sigma_{i=1}^{n}\left[f_{i} \frac{\partial}{\partial x_{i}}\left(a d_{f}^{k-1} g(x)\right)_{j}\right. \\
& \left.-\left(a d_{f}^{k-1} g(x)\right)_{i} \frac{\partial}{\partial x_{i}} f_{j}\right] .
\end{aligned}
$$

In some neighbourhood $N\left(x_{0}\right)$ of a point $x_{0}$, if the matrix,

$$
M=\left[g\left(x_{0}\right), a d_{f} g\left(x_{0}\right), a d_{f}^{2} g\left(x_{0}\right), \ldots, a d_{f}^{n-1} g\left(x_{0}\right)\right]
$$

has a $\operatorname{rank} n$ and $S=\operatorname{span}\left\{g, a d_{f} g, a d_{f}^{2} g, \ldots, a d_{f}^{n-2} g\right\}$ is involutive, then there exists a real valued function $\lambda(x)$ in $N\left(x_{0}\right)$, such that: $L_{g} \lambda(x)=L_{a d_{f} g} \lambda(x)=L_{a d_{f}^{2} g} \lambda(x)=$ $\cdots=L_{a d_{f}^{n-2} g} \lambda(x)=0$, and $L_{a d_{f}^{n-1} g} \lambda(x) \neq 0$ where $L_{F} \lambda(x)$ denotes the Lie derivative of the real valued function $\lambda(x)$ with respect to the vector field $F$. If that happens there exists transformation in $N\left(x_{0}\right)$, given by:

$$
\begin{aligned}
Z & =\left[z_{1}, z_{2}, z_{3}, \ldots, z_{n}\right]^{T}=T(x) \\
& =\left[T_{1}(x), T_{2}(x), \ldots, T_{n}(x)\right]^{T} \\
& =\left[\lambda(x), L_{f} \lambda(x), \ldots, L_{f}^{n-1} \lambda(x)\right]^{T}
\end{aligned}
$$

and

$$
v=L_{f}^{n} \lambda(x)+L_{g} L_{f}^{n-1} \lambda(x) u
$$

that transforms the non-liner system into the linear controllable system: [5-7,11]

$$
\begin{gathered}
\dot{Z}_{1}=Z_{2} \\
\dot{Z}_{2}=Z_{3} \\
\vdots \\
\dot{Z}_{n-1}=Z_{n} \\
\dot{Z}_{n}=v
\end{gathered}
$$


Note: The real valued function $\lambda(x)$ is also known as the output function for the control system.

\section{Problem formulation}

Shimizu-Morioka dynamical system is a chaotic [10,12] system that is algebraically simpler than the Lorentz system but produces qualitatively the same dynamics as Lorentz system in its chaotic regime. The Shimizu Morioka non-linear dynamical system is given by:

$$
\begin{aligned}
& \dot{x_{1}}=x_{2} \\
& \dot{x_{2}}=x_{1}-\beta x_{2}-x_{1} x_{3} \\
& \dot{x_{3}}=-\alpha x_{3}+x_{1}^{2}
\end{aligned}
$$

$\alpha, \beta$ are parameters of the system. For chaos, the values of the parameters are $\alpha=0.375$ and $\beta=0.81$. The system of Eq. (5) can be written as $\dot{x}=f(x)$ where, $x=\left[x_{1}, x_{2}, x_{3}\right]^{T}$, and,

$$
f(x)=\left(\begin{array}{c}
x_{2} \\
x_{1}-\beta x_{2}-x_{1} x_{3} \\
-\alpha x_{3}+x_{1}^{2}
\end{array}\right)
$$

Parametric entrainment control $u\left(x_{1}, x_{2}, x_{3}\right)$ is applied to the parameter $\beta$ in the second equation of the system (5) and one gets,

$\dot{x}=f(x)+g(x) u$

where $g(x)=\left[0,-x_{2}, 0\right]^{T}$ and $u \in \mathbb{R}$.

\section{Control of the chaotic system}

From the previous section,

$$
f(x)=\left(\begin{array}{c}
x_{2} \\
x_{1}-\beta x_{2}-x_{1} x_{3} \\
-\alpha x_{3}+x_{1}^{2}
\end{array}\right), \quad g(x)=\left(\begin{array}{c}
0 \\
-x_{2} \\
0
\end{array}\right)
$$

Lemma 1 For any $x_{0} \in \mathbb{R}^{3} \backslash \operatorname{span}\{(1,0,0),(0,0,1)\} \cup \operatorname{span}$ $\{(0,1,0),(0,0,1)\}$, there exists an open set $N\left(x_{0}\right)$ containing $x_{0}$ where the matrix $M=\left[g\left(x_{0}\right), a d_{f} g\left(x_{0}\right), a d_{f}^{2} g\left(x_{0}\right)\right]$ has rank 3 and $S=\operatorname{span}\left\{g, a d_{f} g\right\}$ is involutive.

Proof Using the method described in the Sect. 2 one gets

$\operatorname{ad}_{f} g(x)=[f, g]=\left(\begin{array}{c}x_{2} \\ x_{1} x_{3}-x_{1} \\ 0\end{array}\right)$

and,

$$
\begin{aligned}
a d_{f}^{2} g(x) & =\left[f, a d_{f} g\right] \\
& =\left(\begin{array}{c}
2 x_{1}-\beta x_{2}-2 x_{1} x_{3} \\
\left(x_{3}-1\right)\left(2 x_{2}+\beta x_{1}\right)+x_{1}^{3}-\alpha x_{1} x_{3} \\
-2 x_{1} x_{2} .
\end{array}\right)
\end{aligned}
$$

So if $x_{1} \neq 0$ and $x_{2} \neq 0$,

$$
\begin{aligned}
& \operatorname{det}(M) \\
& =\left|\begin{array}{ccc}
0 & x_{2} & 2 x_{1}-\beta x_{2}-2 x_{1} x_{3} \\
-x_{2} & x_{1} x_{3}-x_{1} & \left(x_{3}-1\right)\left(2 x_{2}+\beta x_{1}\right)+x_{1}^{3}-\alpha x_{1} x_{3} \\
0 & 0 & -2 x_{1} x_{2}
\end{array}\right| \\
& =-2 x_{1} x_{2}^{3} \neq 0 .
\end{aligned}
$$

therefore, $\operatorname{rank}(M)=3$, which is the order of the system. With the help of (7) and (8) one gets

$$
\begin{aligned}
{\left[g, a d_{f} g\right] } & =\left(\begin{array}{c}
-x_{2} \\
x_{1} x_{3}-x_{1} \\
0
\end{array}\right) \\
& =\frac{2 x_{1}\left(1-x_{3}\right)}{x_{2}} g(x)+(-1) a d_{f} g
\end{aligned}
$$

which shows that $\left[g, a d_{f} g\right]$ belongs to $S=\operatorname{span}\left\{g(x), a d_{f}\right.$ $g(x)\}$. Hence, $S$ is involutive.

Lemma 2 For any thrice differentiable function $\Psi\left(x_{3}\right)$, there exists, a smooth transformation $z=\left[\Psi, L_{f} \Psi, L_{f}^{2} \Psi\right]^{T}$ with a smooth inverse, defined in an open set $N\left(x_{0}\right)$ where $x_{0} \in \mathbb{R}^{3} \backslash \operatorname{span}\{(1,0,0),(0,0,1)\} \cup \operatorname{span}\{(0,1,0),(0,0$, $1)\}$, that reduces system (5) to a linear controllable form.

Proof Since lemma 1 holds, there exists a real valued function $\lambda(x)$ such that $L_{g} \lambda(x)=0$ and $L_{a d_{f}} \lambda(x)=0$ but, $L_{a d_{f}^{2} g} \lambda(x) \neq 0$. Now, $L_{g} \lambda(x)=0$ implies $\frac{\partial \lambda}{\partial x_{2}}=0$, and $L_{a d_{f} g} \lambda(x)=0$ implies $\frac{\partial \lambda}{\partial x_{1}}=0$. Hence $\lambda(x)$ is independent of $x_{1}$ and $x_{2}$ but depends on $x_{3}$. Thus,

$\lambda(x)=\Psi\left(x_{3}\right)$,

where simple calculations yield, $L_{a d_{f}^{2} g} \lambda(x)=-2 x_{1} x_{2} \Psi^{\prime}$ $\left(x_{3}\right) \neq 0$ as $x_{1} \neq 0$ and $x_{2} \neq 0$. With the help (11) one can easily calculate the following Lie derivatives given as

$$
\begin{aligned}
L_{f} \lambda(x)= & \left(x_{1}^{2}-\alpha x_{3}\right) \Psi^{\prime}\left(x_{3}\right) \\
L_{f}^{2} \lambda(x)= & \left(2 x_{1} x_{2}-\alpha\left(x_{1}^{2}-\alpha x_{3}\right)\right) \Psi^{\prime}\left(x_{3}\right) \\
& +\left(x_{1}^{2}-\alpha x_{3}\right)^{2} \Psi^{\prime \prime}\left(x_{3}\right) \\
L_{f}^{3} \lambda(x)= & 2\left[\left(x_{2}-\alpha x_{1}\right) x_{2}+x_{1}\left(x_{1}-\beta x_{2}-x_{1} x_{3}\right)\right. \\
& \left.+\alpha^{2}\left(x_{1}^{2}-\alpha x_{3}\right)\right] \Psi^{\prime}\left(x_{3}\right) \\
& +\left[4 x_{1} x_{2}\left(x_{1}^{2}-\alpha x_{3}\right)\right. \\
& \left.+\left(x_{1}^{2}-\alpha x_{3}\right)\left\{2 x_{1} x_{2}-3 \alpha\left(x_{1}^{2}-\alpha x_{3}\right)\right\}\right] \Psi^{\prime \prime}\left(x_{3}\right) \\
& +\left(x_{1}^{2}-\alpha x_{3}\right)^{3} \Psi^{\prime \prime \prime}\left(x_{3}\right) \\
L_{g} L_{f}^{2} \lambda(x)= & -2 x_{1} x_{2} \Psi^{\prime}\left(x_{3}\right)
\end{aligned}
$$


With the help of (12) the transformation formula (3) takes the form

$$
\begin{aligned}
z & =\left(\begin{array}{l}
z_{1} \\
z_{2} \\
z_{3}
\end{array}\right)=T(x)=\left(\begin{array}{c}
T_{1}(x) \\
T_{2}(x) \\
T_{3}(x)
\end{array}\right)=\left(\begin{array}{c}
\lambda(x) \\
L_{f} \lambda(x) \\
L_{f}^{2} \lambda(x)
\end{array}\right) \\
& =\left(\begin{array}{c}
\Psi\left(x_{3}\right) \\
\left(x_{1}^{2}-\alpha x_{3}\right) \Psi^{\prime}\left(x_{3}\right) \\
\left(2 x_{1} x_{2}-\alpha\left(x_{1}^{2}-\alpha x_{3}\right)\right) \Psi^{\prime}\left(x_{3}\right)+\left(x_{1}^{2}-\alpha x_{3}\right)^{2} \Psi^{\prime \prime}\left(x_{3}\right)
\end{array}\right)
\end{aligned}
$$

Inverse transformation can be calculated from (13) as,

$$
\left(\begin{array}{l}
x_{1} \\
x_{2} \\
x_{3}
\end{array}\right)=x=T^{-1}(z)=\left(\begin{array}{l}
T_{1}^{-1}\left(z_{1}, z_{2}, z_{3}\right) \\
T_{2}^{-1}\left(z_{1}, z_{2}, z_{3}\right) \\
T_{3}^{-1}\left(z_{1}, z_{2}, z_{3}\right)
\end{array}\right)
$$

Controller $u$ is obtained from the formula (3) as:

$u=\frac{1}{L_{g} L_{f}^{2} \lambda(x)}\left[v-L_{f}^{3} \lambda(x)\right]$

These will change the original non-linear system (5) into a linear controllable system:

$$
\begin{aligned}
& \dot{z_{1}}=z_{2} \\
& \dot{z_{2}}=z_{3} \\
& \dot{z_{3}}=v
\end{aligned}
$$

if the new function $v$ is considered linear in $z_{1}, z_{2}$ and $z_{3}$.

Without loss of generality one may take the linear form of $v$ as

$v=a_{1} z_{1}+a_{2} z_{2}+a_{3} z_{3}$

where, $a_{1}, a_{2}, a_{3}$ are real numbers.

Theorem 1 (Stabilization at a point) The controller $v=a_{1} z_{1}+a_{2} z_{2}+a_{3} z_{3}$ stabilizes the equilibrium point $z=(0,0,0)$ iff $a_{1}<0, a_{2}<0, a_{3}<0$ and $a_{1}+a_{2} a_{3}>0$.

Proof With the introduction of the controller $v=a_{1} z_{1}$ $+a_{2} z_{2}+a_{3} z_{3}$ in the system (16), it takes the form $\dot{Z}=A Z$, where

$Z=\left(\begin{array}{c}z_{1} \\ z_{2} \\ z_{3}\end{array}\right), \quad A=\left(\begin{array}{ccc}0 & 1 & 0 \\ 0 & 0 & 1 \\ a_{1} & a_{2} & a_{3}\end{array}\right)$

Equilibrium point $(0,0,0)$ of this system will be stable iff all eigenvalues of the matrix $A$ have negative real parts. Characteristic equation of the matrix $\mathrm{A}$ is $\lambda^{3}-a_{3} \lambda^{2}-a_{2} \lambda-a_{1}=$ 0 . From Routh-Hurwitz criterion, the roots of the above equation will have negative real parts iff $a_{1}<0, a_{2}<0, a_{3}<0$ and $a_{2} a_{3}+a_{1}>0$. Hence the result follows.
Theorem 2 (Stabilization onto a limit cycle-Hopf bifurcation) The controller $v=a_{1} z_{1}+a_{2} z_{2}+a_{3} z_{3}$ stabilizes the system on to a stable limit cycle iff $a_{1}<0, a_{2}<0, a_{3}<$ 0 and $a_{1}+a_{2} a_{3}=0$.

Proof The system (16) with the linear controller $v$ is investigated for the possibility of a Hopf bifurcation. The system being three dimensional, the condition for generation of stable limit cycles through Hopf bifurcation can easily be determined by imposing the restrictions:

(i) A negative real eigenvalue, and,

(ii) A conjugate pair of purely imaginary eigenvalues,

on the characteristic equation. If $-p_{1}$ and $\pm i p_{2}$ be the roots of the characteristic equation of the matrix $A$ in Theorem 1 with $p_{1}>0$ and $p_{2}$ being real, one can easily have: $a_{1}=$ $-p_{1} p_{2}^{2}, a_{2}=-p_{2}^{2}$ and $a_{3}=-p_{1}$. Now it is easy to observe that $a_{1}<0, a_{2}<0, a_{3}<0$ and $a_{2} a_{3}+a_{1}=0$.

Theorem 3 The system (6) with an output function $\Psi\left(x_{3}\right)$ becomes controlled when the control loop is closed with control input

$$
\begin{aligned}
u= & \frac{1}{2 x_{1} x_{2}}\left[2 x_{2}\left(x_{2}-\alpha x_{1}\right)+2 x_{1}\left(x_{1}-\beta x_{2}-x_{1} x_{3}\right)\right. \\
& +\alpha^{2}\left(x_{1}^{2}-\alpha x_{3}\right)+3\left(x_{1}^{2}-\alpha x_{3}\right)\left\{2 x_{1} x_{2}\right. \\
& \left.-\alpha\left(x_{1}^{2}-\alpha x_{3}\right)\right\} \frac{\Psi^{\prime \prime}\left(x_{3}\right)}{\Psi^{\prime}\left(x_{3}\right)} \\
& +\left(x_{1}^{2}-\alpha x_{3}\right)^{3} \frac{\Psi^{\prime \prime \prime}\left(x_{3}\right)}{\Psi^{\prime}\left(x_{3}\right)}-\left\{a_{2}\left(x_{1}^{2}-\alpha x_{3}\right)\right. \\
& \left.+a_{3}\left(\alpha^{2} x_{3}-\alpha x_{1}^{2}+2 x_{1} x_{2}\right)\right\} \\
& \left.-a_{1} \frac{\Psi\left(x_{3}\right)}{\Psi^{\prime}\left(x_{3}\right)}-a_{3}\left(x_{1}^{2}-\alpha x_{3}\right)^{2} \frac{\Psi^{\prime \prime}\left(x_{3}\right)}{\Psi^{\prime}\left(x_{3}\right)}\right]
\end{aligned}
$$

Proof Using (12) and (15) and the assumed linear form of $v$, it is easy to obtain $u$ in the above form through some pretty straightforward but laborious calculations.

Now the problem is studied for the particular forms of $\Psi\left(x_{3}\right)$

\subsection{Case I (Linear output)}

Theorem 4 A linear output $\Psi\left(x_{3}\right)=x_{3}-x_{g}$ and control law

$$
\begin{aligned}
u= & \frac{1}{2 x_{1} x_{2}}\left[2 x_{2}\left(x_{2}-\alpha x_{1}\right)+2 x_{1}\left(x_{1}-\beta x_{2}-x_{1} x_{3}\right)\right. \\
& +\alpha^{2}\left(x_{1}^{2}-\alpha x_{3}\right)-a_{1}\left(x_{3}+k\right)-a_{2}\left(x_{1}^{2}-\alpha x_{3}\right) \\
& \left.-a_{3}\left(\alpha^{2} x_{3}-\alpha x_{1}^{2}+2 x_{1} x_{2}\right)\right]
\end{aligned}
$$


stabilizes system (5) to the control goal $\vec{x}_{g}=\left(\sqrt{\alpha x_{g}}, 0, x_{g}\right)^{T}$ where $x_{g}$ is the parameter that determines the control goal.

Proof Let $\Psi\left(x_{3}\right)$ be taken as $x_{3}+k$, i.e. $\lambda(x)=\Psi\left(x_{3}\right)=$ $x_{3}+k$, where $k$ is arbitrary constant to be determined later. Controlling the system (16) to the origin and changing the values of $k$ one can control $x$ to the control goal $x_{g}$. In this case control law takes the form

$$
\begin{aligned}
u= & \frac{1}{2 x_{1} x_{2}}\left[2 x_{2}\left(x_{2}-\alpha x_{1}\right)+2 x_{1}\left(x_{1}-\beta x_{2}-x_{1} x_{3}\right)\right. \\
& +\alpha^{2}\left(x_{1}^{2}-\alpha x_{3}\right)-a_{1}\left(x_{3}+k\right)-a_{2}\left(x_{1}^{2}-\alpha x_{3}\right) \\
& \left.-a_{3}\left(\alpha^{2} x_{3}-\alpha x_{1}^{2}+2 x_{1} x_{2}\right)\right]
\end{aligned}
$$

for the transformation (13),

$$
\left(\begin{array}{l}
z_{1} \\
z_{2} \\
z_{3}
\end{array}\right)=\left(\begin{array}{c}
x_{3}+k \\
x_{1}^{2}-\alpha x_{3} \\
2 x_{1} x_{2}-\alpha x_{1}^{2}+\alpha^{2} x_{3}
\end{array}\right)
$$
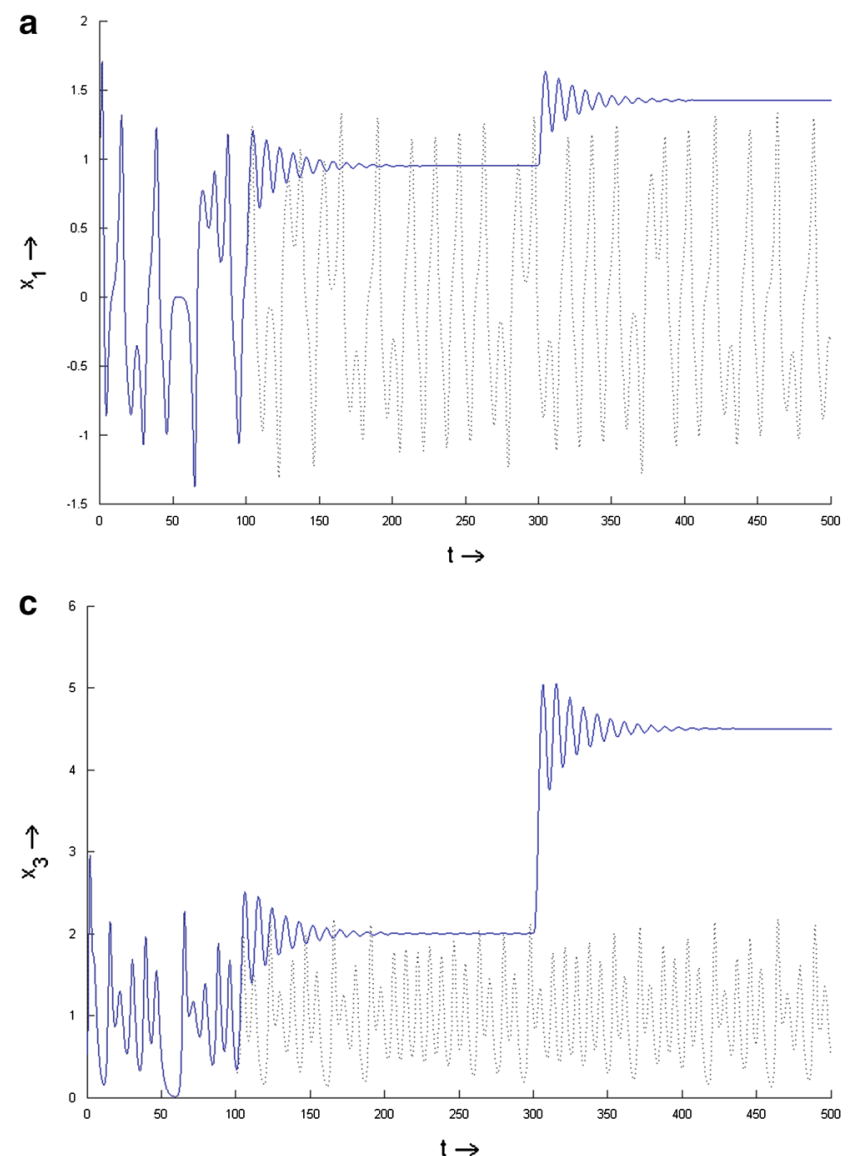

The inverse transformation obtained from (19) is,

$$
\begin{aligned}
\left(\begin{array}{l}
x_{1} \\
x_{2} \\
x_{3}
\end{array}\right) & =T^{-1}(z)=\left(\begin{array}{c}
T_{1}^{-1}(z) \\
T_{2}^{-1}(z) \\
T_{3}^{-1}(z)
\end{array}\right) \\
& =\left(\begin{array}{c}
\sqrt{z_{2}+\alpha\left(z_{1}-k\right)} \\
\frac{z_{3}+\alpha z_{2}}{2 \sqrt{z_{2}+\alpha\left(z_{1}-k\right)}} \\
z_{1}-k
\end{array}\right)
\end{aligned}
$$

Since the feedback control law stabilizes the equilibrium point of (16), then using (20) and changing the value of $k$ one can control $x_{3}$ to the goal $x_{g}$ and the variation of $k$ is given by the formula

$k=-x_{g}$

As $x_{3}$ goes to the goal $x_{g}$, the state vector $x$ goes to

$$
\left(\begin{array}{c}
\sqrt{\alpha x_{g}} \\
0 \\
x_{g}
\end{array}\right)
$$
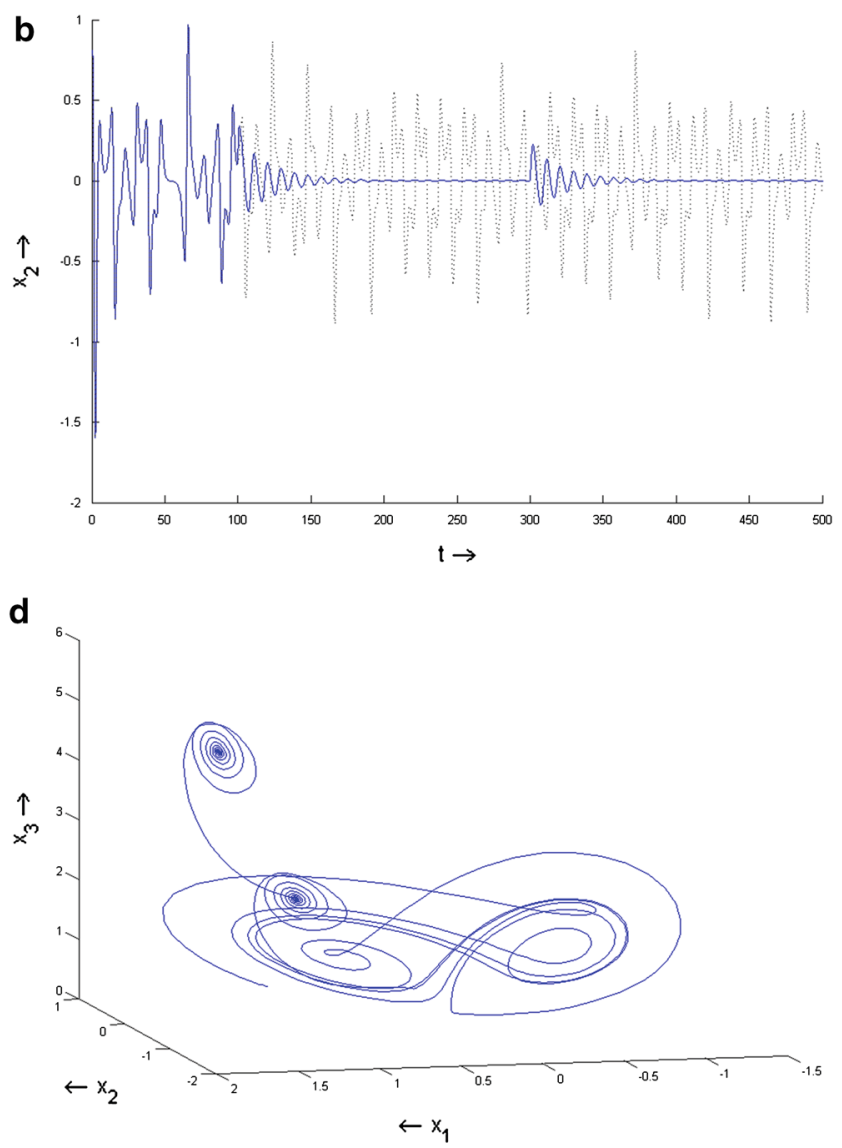

Fig. 1 Stabilization of Shimizu-Morioka chaotic system to a point for a linear observer with controller parameters $a_{1}=-0.15, a_{2}=-0.5, a_{3}=$ $-0.4$ 


\subsubsection{Numerical simulation results}

For numerical simulation the values of the system parameters are taken as $\alpha=0.375, \beta=0.81$ so that Shimizu Morioka(SM) system is in the chaotic regime. The system is integrated with initial conditions $x_{1}(0)=0.9, x_{2}(0)=$ $0.8, x_{3}(0)=0.2$. The control is activated at time $t=100$ with control goal $x_{g}=2$ and at time $t=300$, control goal is modified to $x_{g}=4.5$ instantly. The time response of the controlled system (solid line) and the original chaotic system (dotted line) are analyzed under these conditions by plotting them together. Controller parameters $a_{1}, a_{2}, a_{3}$ are modified to illustrate the different results obtained analytically. Simulation work also provides some interesting insights, particularly in the case of stabilization of the limit cycle.

For Fig. 1, $a_{1}=-0.15, a_{2}=-0.5, a_{3}=-0.4$ and according to Theorem 1 , the system attains the control goal in finite time. The initial fluctuations in the state space on activation of control or on changing the control goal can be critically damped with values of $a_{1}$ much closer to 0 . The figures also establish robustness of the control scheme towards goal fluctuations provided the control goal lies within a reachable set. The fluctuations are of no significance to control action as the system always reaches the goal in finite time.

Figure 2 illustrate the interesting case of stable limit cycles arising in the controlled system, as noted in Theorem 2. Choosing $a_{1}=-0.2, a_{2}=-0.5, a_{3}=-0.4$, it is possible to stabilize the system trajectories onto a limit cycle. As opposed to the commonly observed situation of stabilizing the system to an equilibrium point through feedback linearization, we explore the possibility of stabilizing the system to a periodic state. The phase diagram also shows that goal fluctuation doesn't disturb this property of the controlled system. The only observable effect of goal fluctuations is in the amplitude of the limit cycles which tend to get smaller for larger goals. But the period of the limit cycle remains constant.

Another point of concern is the effect of changing $a_{2}$. Hence for Fig. 3a, parameter values $a_{1}=-0.1, a_{2}=$ $-0.3, a_{3}=-0.4$ are used in accordance with Theorem 1 . As compared to Fig. 1, $a_{2}$ has witnessed a marked decrease. As a result, the system still approaches the control goal but the damping effect is much less that leads to much more pronounced fluctuations in the state space. For Fig. 3b,
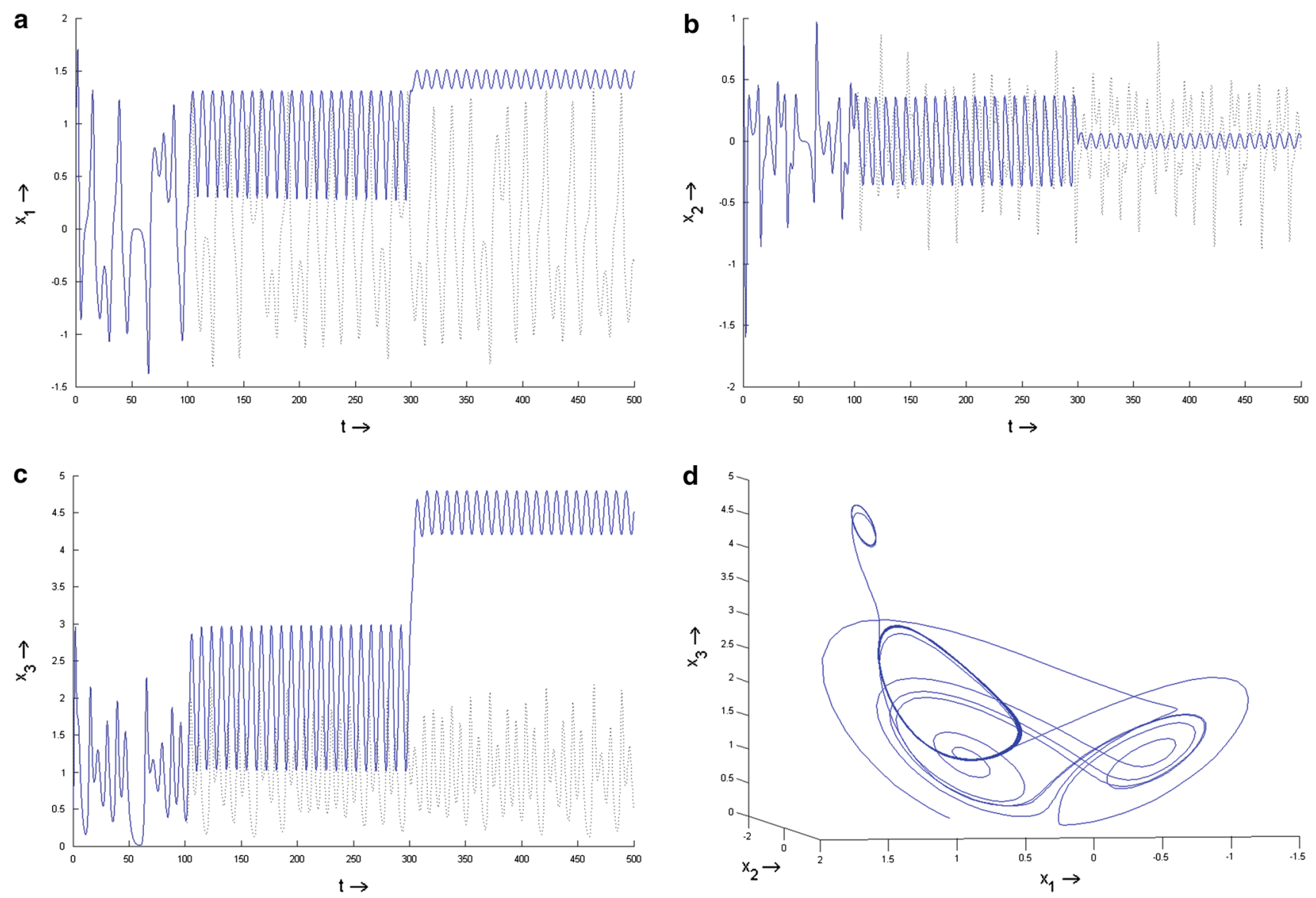

Fig. 2 Stabilization of Shimizu-Morioka chaotic system onto a limit cycle for a linear observer with controller parameters $a_{1}=-0.2, a_{2}=$ $-0.5, a_{3}=-0.4$ 

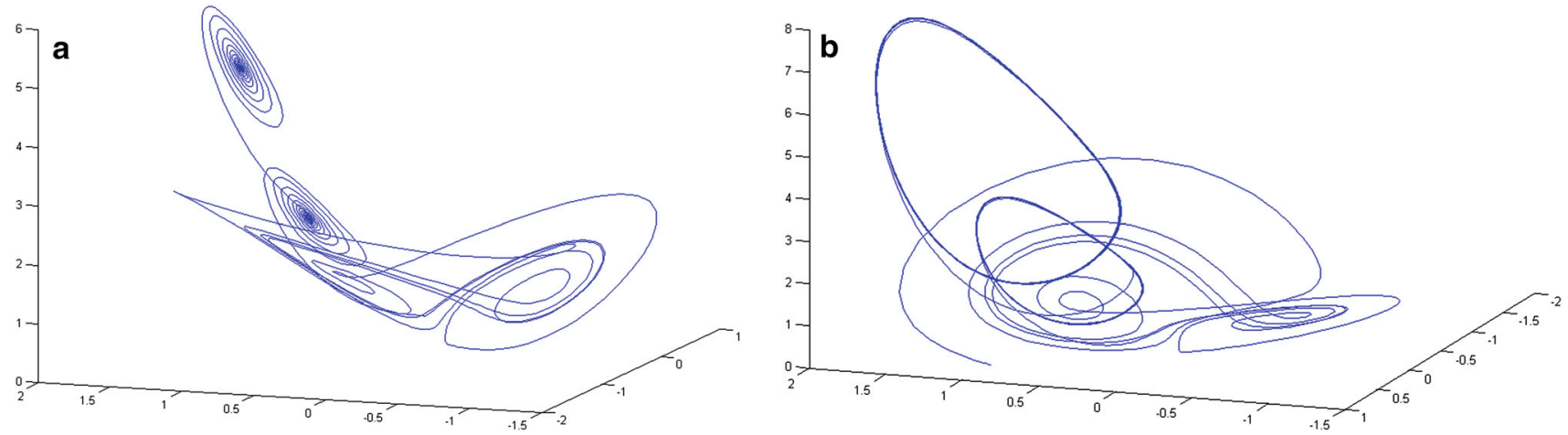

Fig. 3 Phase diagrams for stabilization of the system to a point and to a limit cycle for parameter choice $\mathbf{a}$, left $a_{1}=-0.1, a_{2}=-0.3, a_{3}=-0.4$, b, right $a_{1}=-0.12, a_{2}=-0.3, a_{3}=-0.4$

$a_{1}=-0.12, a_{2}=-0.3, a_{3}=-0.4$ is used. It again generates stable limit cycles as claimed in Theorem 2. In contrast to Fig. 2, the amplitude of the limit cycle around the goal corresponding to $x_{g}=4.5$ is much larger. Further experimentations reveal that the values of $a_{1}$ and $a_{2}$ are instrumental in deciding the amplitude of the limit cycle. Analogous results are observed for the variation of parameter $a_{3}$ but the effects are not as sharp as observed for the variation of $a_{1}$ and $a_{2}$.

Parameter variations reveal that larger parameter choices lets us avoid transient fluctuations on control activation or goal update. However, such large control force implies that the interesting feature of limit cycles would go unobserved. Small controller parameters are more suited for stabilizing the system onto a limit cycle. Exploration of the parameter space numerically indicates that dynamics of the controlled system cannot get anymore complex than this. Thus, we can conclude that chaos has been successfully controlled in the SM system.

\subsection{Case II (Non-linear output)}

Theorem 5 A non-linear output $\lambda(x)=\Psi\left(x_{3}\right)=x_{3}^{2}+x_{3}$ $+\mu$ and control law

$$
\begin{aligned}
u= & \frac{1}{2 x_{1} x_{2}\left(2 x_{3}+1\right)}\left[6\left(x_{1}^{2}-\alpha x_{3}\right)\left(2 x_{1} x_{2}-\alpha x_{1}^{2}+\alpha^{2} x_{3}\right)\right. \\
& \left.-2 a_{3}\left(x_{1}^{2}-\alpha x_{3}\right)^{2}-a_{1}\left(x_{3}^{2}+x_{3}+\mu\right)\right] \\
& +\frac{1}{2 x_{1} x_{2}}\left[2 x_{2}\left(x_{2}-\alpha x_{1}\right)+2 x_{1}\left(x_{1}-\beta x_{2}-x_{1} x_{3}\right)\right. \\
& +\alpha^{2}\left(x_{1}^{2}-\alpha x_{3}\right)-a_{2}\left(x_{1}^{2}-\alpha x_{3}\right) \\
& \left.-a_{3}\left(\alpha^{2} x_{3}-\alpha x_{1}^{2}+2 x_{1} x_{2}\right)\right]
\end{aligned}
$$

stabilizes system (5) to the control goal $\vec{x}_{g}=\left( \pm \sqrt{\alpha x_{g}}, 0, x_{g}\right)^{T}$ where $\mu=\frac{1}{4}\left[1-\left(1+2 x_{g}\right)^{2}\right] \leq \frac{1}{4}$ and $x_{g}$ is the parameter that determines the control goal $\vec{x}_{g}$.
Proof In this case $\Psi\left(x_{3}\right)$ is taken as $\lambda(x)=\Psi\left(x_{3}\right)=x_{3}^{2}+$ $x_{3}+\mu$ where $\mu$ is arbitrary constant whose value is chosen according to the control goal. The controller in this case is given by,

$$
\begin{aligned}
u= & \frac{1}{2 x_{1} x_{2}\left(2 x_{3}+1\right)}\left[6\left(x_{1}^{2}-\alpha x_{3}\right)\left(2 x_{1} x_{2}-\alpha x_{1}^{2}+\alpha^{2} x_{3}\right)\right. \\
& \left.-2 a_{3}\left(x_{1}^{2}-\alpha x_{3}\right)^{2}-a_{1}\left(x_{3}^{2}+x_{3}+\mu\right)\right] \\
& +\frac{1}{2 x_{1} x_{2}}\left[2 x_{2}\left(x_{2}-\alpha x_{1}\right)+2 x_{1}\left(x_{1}-\beta x_{2}-x_{1} x_{3}\right)\right. \\
& +\alpha^{2}\left(x_{1}^{2}-\alpha x_{3}\right)-a_{2}\left(x_{1}^{2}-\alpha x_{3}\right) \\
& \left.-a_{3}\left(\alpha^{2} x_{3}-\alpha x_{1}^{2}+2 x_{1} x_{2}\right)\right]
\end{aligned}
$$

which is obtained for the transformation formula,

$$
\begin{aligned}
& \left(\begin{array}{l}
z_{1} \\
z_{2} \\
z_{3}
\end{array}\right)=Z=T(x)=\left(\begin{array}{l}
T_{1}(x) \\
T_{2}(x) \\
T_{3}(x)
\end{array}\right) \\
& =\left(\begin{array}{c}
x_{3}^{2}+x_{3}+\mu \\
\left(2 x_{3}+1\right)\left(x_{1}^{2}-\alpha x_{3}\right) \\
\left(2 x_{3}+1\right)\left(2 x_{1} x_{2}-\alpha x_{1}^{2}+\alpha^{2} x_{3}\right)+2\left(x_{1}^{2}-\alpha x_{3}\right)^{2}
\end{array}\right)
\end{aligned}
$$

The inverse transformation of (23) is given by,

$$
\begin{aligned}
& \left(\begin{array}{l}
x_{1} \\
x_{2} \\
x_{3}
\end{array}\right)=T^{-1}(z)=\left(\begin{array}{c}
T_{1}^{-1}(z) \\
T_{2}^{-1}(z) \\
T_{3}^{-1}(z)
\end{array}\right) \\
& =\left(\begin{array}{c}
A \\
\left(z_{3}+\alpha z_{2}-\frac{2 z_{2}^{2}}{1-4 \mu+4 z_{1}}\right) \frac{1}{2 A B} \\
-\frac{1 \pm B}{2}
\end{array}\right)
\end{aligned}
$$

where $A^{2}=-\frac{\alpha}{2} \pm\left(\frac{z_{2}}{B^{2}}+\frac{\alpha}{2}\right) B$, and $B^{2}=1-4\left(\mu-z_{1}\right)$. Feedback control law (22) stabilizes the equilibrium point of 

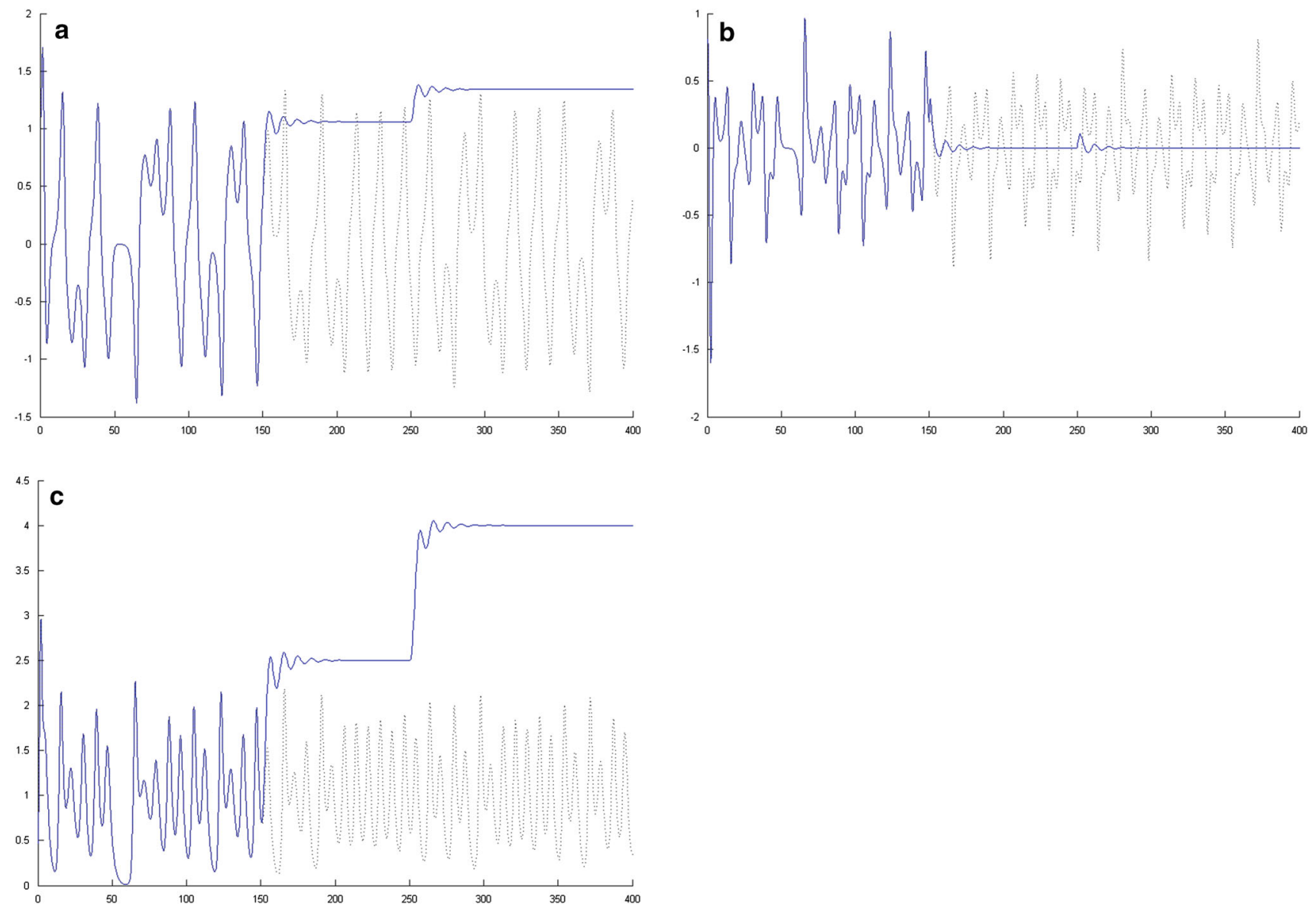

Fig. 4 Control of the system in presence of non-linear observer with $a_{1}=-0.1, a_{2}=-0.5, a_{3}=-0.4$
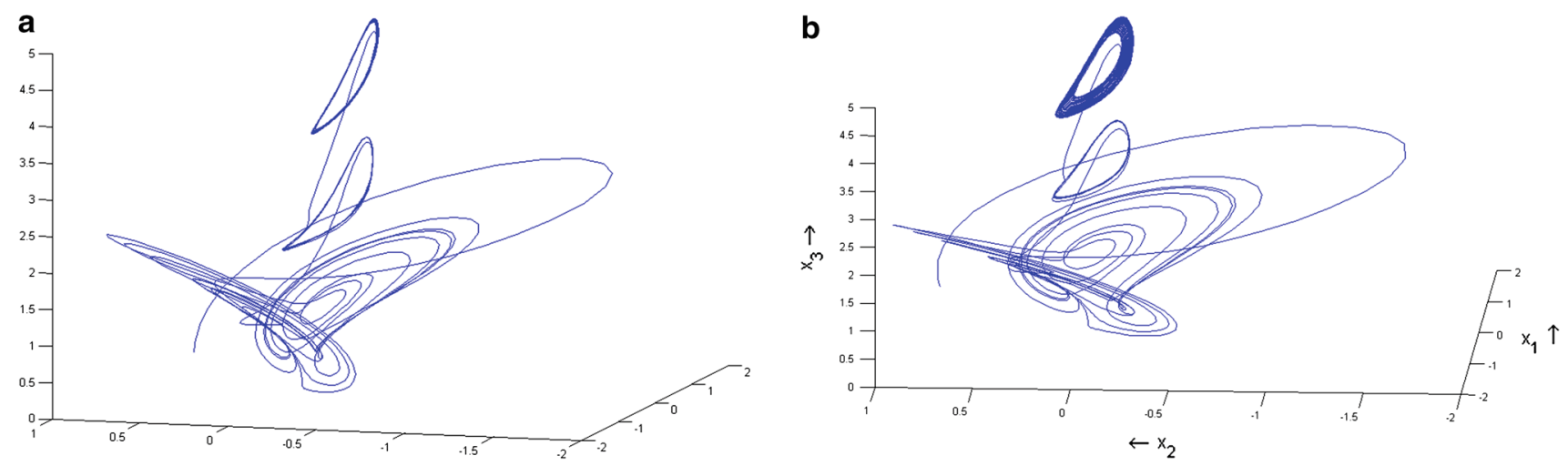

Fig. 5 Stabilization onto limit cycles for non-linear observer with parameters: a, left $a_{1}=-0.2, a_{2}=-0.5, a_{3}=-0.4$, b, right $a_{1}=$ $-0.15, a_{2}=-0.5, a_{3}=-0.3$

(16). Using (24) and changing the value of $\mu$ one can control $x_{3}$ to the control goal $x_{g}$ and the variation of $\mu$ is given by the formula

$$
\mu=\frac{1}{4}\left[1-\left(1+2 x_{g}\right)^{2}\right]
$$

For real goal $x_{g}$ one must take $\mu \leq \frac{1}{4}$. If $x_{3}$ goes to the goal $x_{g}$, the state vector $x$ goes to the point

$$
\left(\begin{array}{c} 
\pm \sqrt{\alpha x_{g}} \\
0 \\
x_{g}
\end{array}\right)
$$




\subsubsection{Numerical simulation results}

For numerical simulation, we again choose $\alpha=0.375$ and $\beta=0.81$. The initial conditions are kept $x_{1}(0)=$ $0.9, x_{2}(0)=0.8, x_{3}(0)=0.2$ again. The control is activated at time $t=150$ with control goal $x_{g}=2.5$ and at time $t=250$, control goal is modified to $x_{g}=4$ instantly. The time response of the controlled system (solid line) and the original chaotic system (dotted line) are analyzed under these conditions by plotting them together. Controller parameters $a_{1}, a_{2}, a_{3}$ are modified to illustrate the stabilization of the system both onto an equilibrium point and on to a stable limit cycle.

For Fig. 4, $a_{1}=-0.1, a_{2}=-0.5, a_{3}=-0.4$ and the system stabilizes on to the equilibrium point in finite time. Notably, though the controller parameters $a_{i}$ are not very much different from that in Sect. 4.1.1, the fluctuation in state space is not so pronounced. This is mainly due to the quadratic observer/ output function choice. Choosing $a_{1}=$ $-0.2, a_{2}=-0.5, a_{3}=-0.4$, the system is stabilized onto a stable limit cycle in Fig. 5a. Here the time amplitude of the limit cycles for two different $x_{g}$ is comparable.

Figure $5 \mathrm{~b}$ was drawn with $a_{1}=-0.15, a_{2}=-0.5, a_{3}=$ -0.3 . The limit cycle around $x_{g}=2.5$ is of no special significance. But interestingly, the trajectories in phase space keep oscillating in the neighbourhood of the limit cycle for $x_{g}=4$. The trajectories settle down onto the trajectory extremely slowly in spite of parameters satisfying Theorem 2. Thus, this remains an interesting observation that is unique to the case of non-linear observer.

Acknowledgments The authors acknowledge the anonymous reviewers for their insightful comments and suggestions.

\section{References}

1. Andrievskii BR, Fradkov AL et al (2003) Control of chaos: methods and applications. Autom Remote Control 64(5):713

2. Babloyantz A, Krishchenko AP, Nosov A (1997) Analysis and stabilization of nonlinear chaotic systems. Comput Math Appl 34:355-368

3. Chen LQ, Liu YZ (1999) A modied exact linearization control for chaotic oscillators. Nonlin Dyn 20:309-317

4. Yu XH (1997) Controlling chaos using input-output linearization approach. Int J Bifurcat Chaos 7:1659-1664

5. Tsagas GR, Mazumdar HP et al (2000) On the control of a dynamical system by a linearization method via Lie Algebra. Rev Bull Cal Math Soc 8(1,2):25-32

6. Liqun C, Yanzhu L (1998) Control of Lorenz chaos by the exact linearization. Appl Math Mech 19(1):67-73

7. Alvarez-Gallegos J (1994) Non-linear regulation of a Lorenz system by feedback linearization techniques. Dyn Control 4:272-289

8. Islam N, Mazumdar HP, Das A (2009) On the stability and control of Shimuzu Morioka system of differential equations. Diff Geom Dyn Syst 11:135-143

9. Islam N, Islam B, Mazumdar HP et al (2011) Generalised chaos synchronization of unidirectionally coupled Shimizu Morioka dyamical system. Diff Geom Dyn Syst 13:114-119

10. Shimizu T, Moroika N (1980) On the bifurcation of a symmetric limit cycle to an asymmetric one in a single model. Phys Lett A 76:201-204

11. Shinbrot T, Grebogi C, Ott E, Yorkee JA (1993) Using small perturbation to control chaos. Nature 363:411-474

12. Islam M, Islam B, Islam N (2013) Rate estimation of identical synchronization by designing controllers. J Math 8. doi:10.1155/ 2013/590462 